\title{
Dielectric Properties of 3D Printed Polylactic Acid
}

\author{
Claudius Dichtl, Pit Sippel, and Stephan Krohns \\ Experimental Physics V, Center for Electronic Correlations and Magnetism, University of Augsburg, 86159 Augsburg, Germany
}

Correspondence should be addressed to Stephan Krohns; stephan.krohns@physik.uni-augsburg.de

Received 3 April 2017; Revised 30 May 2017; Accepted 18 June 2017; Published 17 July 2017

Academic Editor: Marino Lavorgna

Copyright (C) 2017 Claudius Dichtl et al. This is an open access article distributed under the Creative Commons Attribution License, which permits unrestricted use, distribution, and reproduction in any medium, provided the original work is properly cited.

\begin{abstract}
3D printers constitute a fast-growing worldwide market. These printers are often employed in research and development fields related to engineering or architecture, especially for structural components or rapid prototyping. Recently, there is enormous progress in available materials for enhanced printing systems that allow additive manufacturing of complex functional products, like batteries or electronics. The polymer polylactic acid (PLA) plays an important role in fused filament fabrication, a technique used for commercially available low-budget 3D printers. This printing technology is an economical tool for the development of functional components or cases for electronics, for example, for lab purposes. Here we investigate if the material properties of "as-printed" PLA, which was fabricated by a commercially available 3D printer, are suitable to be used in electrical measurement setups or even as a functional material itself in electronic devices. For this reason, we conduct differential scanning calorimetry measurements and a thorough temperature and frequency-dependent analysis of its dielectric properties. These results are compared to partially crystalline and completely amorphous PLA, indicating that the dielectric properties of "as-printed" PLA are similar to the latter. Finally, we demonstrate that the conductivity of PLA can be enhanced by mixing it with the ionic liquid "trihexyl tetradecyl phosphonium decanoate." This provides a route to tailor PLA for complex functional products produced by an economical fused filament fabrication.
\end{abstract}

\section{Introduction}

Additive manufacturing, also named 3D printing or rapid prototyping, moved into various markets due to developments of enhanced printing techniques, enabling many different materials to be printed. The possible applications are numerous, such as titanium scaffolds for orthopaedic implants [1], complex biomedical devices [2], optic components [3], lab-on-a-chip devices [4], and aerial vehicle wing structures [5], to name but a few recent developments. Besides the fast-growing markets for additive manufactured biomaterials and engineered structures, 3D printing also opens a cheap and simple route to produce individual, customised components for scientific use [6-9]. Fused filament fabrication [10] is a low-budget $3 \mathrm{D}$ printing technique often using polymers, especially thermoplastics, as filaments. Biodegradable polylactic acid (PLA) is an example of a polymer that is appropriate for this purpose [11]. The mechanical properties of PLA are already described in detail in [12-14] as well as the references therein. However, for applications as a component in an electrical measurement setup or even as a functional material itself in an electronic device [15], the electric and dielectric properties of PLA [16-20] in the "as-printed" state are rarely investigated.

In this study we focus on the dielectric properties of a commercially available PLA filament, which is processed via fused filament fabrication into an "as-printed" state. The results are compared to PLA in a pure amorphous state as well as to a semicrystalline phase. The latter is achieved by slowly cooling (approximately $0.2 \mathrm{~K} / \mathrm{min}$ ) from a temperature above the melting point of about $430 \mathrm{~K}$ down to a temperature below the glass transition temperature of about $331 \mathrm{~K}$. The purely amorphous state is attained by cooling faster than $10 \mathrm{~K} / \mathrm{min}$ [21]. The melting, the glass transition, and the (cold) crystallization are investigated via differential scanning calorimetry (DSC). The dielectric spectra show that "asprinted" PLA is in a state mimicking the amorphous phase rather than the semicrystalline one. During printing PLA is deposited on a plate or the previously printed material, both at room temperature. This leads to rapid cooling. When the next layers are printed, the underlying material is reheated slightly, resulting in a complex thermal history. Thus, slight 
deviations in melting energies, onset temperatures, and changes in dielectric relaxations are observed, pointing towards a complex short-range order of polymer strings within the amorphous phase [22]. The mesoscopic structure [22] of "as-printed" PLA is most likely influenced by the geometry of the nozzle and the feeding mechanism of the $3 \mathrm{D}$ printer.

To develop functional materials for this printing technology, for example, for electronics, modifying the conductivity of the insulating filament can be an appropriate route. Park et al. $[23,24]$ demonstrated homogenous mixing of PLA with the ionic liquid, trihexyl tetradecyl phosphonium decanoate (THTPT DE). They investigated the physical properties and the degradation of this mixture. There exist innumerable ILs especially taking into account mixtures of various ILs. It seems random to utilize THTPT DE to produce PLA blends. However, it turned out ([24] and references therein) that the type of the cation has a significant impact on the degree of dispersion. Long-chain phosphonium-based (THTPT) ILs show improved dispersion compared to ILs of lower molecular weight giving a starting point for a thorough analysis of suitable ILs for PLA blends. In addition, the used IL has to be prepared in a solution mixable with dissolved PLA; in the present case we dissolved both in chloroform. In general, using ionic liquid as mixing material is a challenging task, as this class of material exhibits manifold chemical compositions. Ionic liquids are salts that are liquid at ambient temperatures offering beneficial properties, for example, low volatility and high-temperature stability [25]. When ionic liquids are used as plasticizers, the properties of a polymer can improve, compared to one with a common molecular plasticizer [24, 26]. Moreover, the ionic nature of these liquids introduces charge carriers, enhancing the conductivity of polymers. Within the scope of this manuscript, we show, following the suggestions of Park et al. [23, 24], that the dielectric properties of PLA are improved by homogenously mixing it with the IL THTPT DE. The achieved result of dielectric spectroscopy allows analysing the conductivity of the mixture, which we performed in a temperature range from 80 to $420 \mathrm{~K}$.

\section{Experimental Details}

The PLA material was prepared from a filament, as described below. The mixture of PLA and THTDP DE was synthesized following the route of Park et al. [23, 24]. For the material analysis we performed DSC and dielectric spectroscopy, which was done in the frequency range below a gigahertz.

2.1. Raw Material and Material Conditions. MakerBot ${ }^{\mathrm{TM}}$ supplied the commercially available PLA in the form of a filament for the employed 3D printer (Makerbot, Replicator 2). Due to the industrial fabrication process the PLA can contain unknown additives. Additionally the stereoisomeric composition (d,1-lactide) is not known. However, the same filament was used for all samples. DSC measurements and dielectric spectroscopy have been conducted on PLA in three different material conditions. In the following they will be designated as "as-printed"; "amorphous"; and "crystalline." For the "asprinted" condition, the $3 \mathrm{D}$ printer was used, heating the PLA to a temperature of approximately $530 \mathrm{~K}$ before extrusion. The samples have been printed in the required geometry with no subsequent heat treatment. The finest resolution settings of the $3 \mathrm{D}$-printer have been used, resulting in a thickness of about $100 \mu \mathrm{m}$ of each deposited layer. For PLA in "amorphous" and "crystalline" state the filament was first heated above the melting point of PLA (about $430 \mathrm{~K}$ ) [21] with subsequent cooling to room temperature at different rates. For the "crystalline" sample the cooling rate was $0.2 \mathrm{~K} / \mathrm{min}$ [26]. To reach a maximum degree of crystalline phase, the sample was tempered for $10 \mathrm{~min}$ at $383 \mathrm{~K}$. To receive a sample in the "amorphous" condition it has to be cooled at a rate greater than $10 \mathrm{~K} / \mathrm{min}$ [21]. The sample for the dielectric measurements was cooled down in a plate-like capacitor at an average rate of approximately $50 \mathrm{~K} / \mathrm{min}$. The sample for the DSC-measurement was cooled in the DSC at a constant rate of $40 \mathrm{~K} / \mathrm{min}$. Both cooling rates are sufficient for the pure "amorphous" state, which is confirmed by the DSC-analysis.

For the PLA/IL mixture we used PLA pellets (NatureWorks LLC), which were dissolved in chloroform (SigmaAldrich, 99\% concentration). After total dilution of the PLA, trihexyl tetradecyl phosphonium decanoate (IoLiTec) was added and the mixture was stirred for $12 \mathrm{~h}$. The THTDP DE itself was dried in advance for $24 \mathrm{~h}$ in vacuum to prevent impurities of residual water [27]. Thin plate-like samples of the PLA/IL mixture were synthesized using the solventcasting method on a Teflon plate. Finally, the samples were dried again for $24 \mathrm{~h}$ at $333 \mathrm{~K}$ in a vacuum chamber. In addition a pure PLA sample was prepared.

\subsection{Differential Scanning Calorimetry. Performing DSC-} analysis of all samples allowed investigating the transition temperatures for melting and crystallization, as well as the enthalpy of these transitions and the glass-temperatures. The heat-flux in or out, respectively, of the sample is measured as a function of temperature, while the sample is cooled or heated at a predefined rate. The samples were sealed in aluminum pans and power compensating DSC 8500 (Perkin Elmer), and heat-flux DSC 204 F1 (NETZSCH) setups were used.

2.3. Dielectric Spectroscopy. Dielectric measurements were used to determine the dielectric constant $\varepsilon^{\prime}$, dielectric loss $\varepsilon^{\prime \prime}$, and conductivity $\sigma^{\prime}$ as function of both temperature and frequency. Measurements in the frequency range from $1 \mathrm{~Hz}$ to $10 \mathrm{MHz}$ were performed using a Novocontrol AlphaAnalyser and in the frequency range from $1 \mathrm{MHz}$ to $3 \mathrm{GHz}$ using an Agilent Impedance Analyser E4991A for cooling and heating nitrogen gas cryostat (Novocontrol Quatro) was deployed to cover the temperature range from $160 \mathrm{~K}$ up to $420 \mathrm{~K}$ and a closed-cycle refrigerator was employed for the temperature range from $80 \mathrm{~K}$ to $300 \mathrm{~K}$. For the "as-printed" sample a disc with a diameter of $5 \mathrm{~mm}$ and thickness of $300 \mu \mathrm{m}$ was printed. The surfaces were polished by abrasive silicon carbide paper. For a plate-like capacitor the bottom and top faces of the sample were covered with conductive silver paint. The "crystalline" and "amorphous" samples have been analysed in capacitors, which are designed for liquid samples (diameter: $12 \mathrm{~mm}$, thickness $50 \mu \mathrm{m}$ ), as both samples had to be heated above the melting point during the sample 


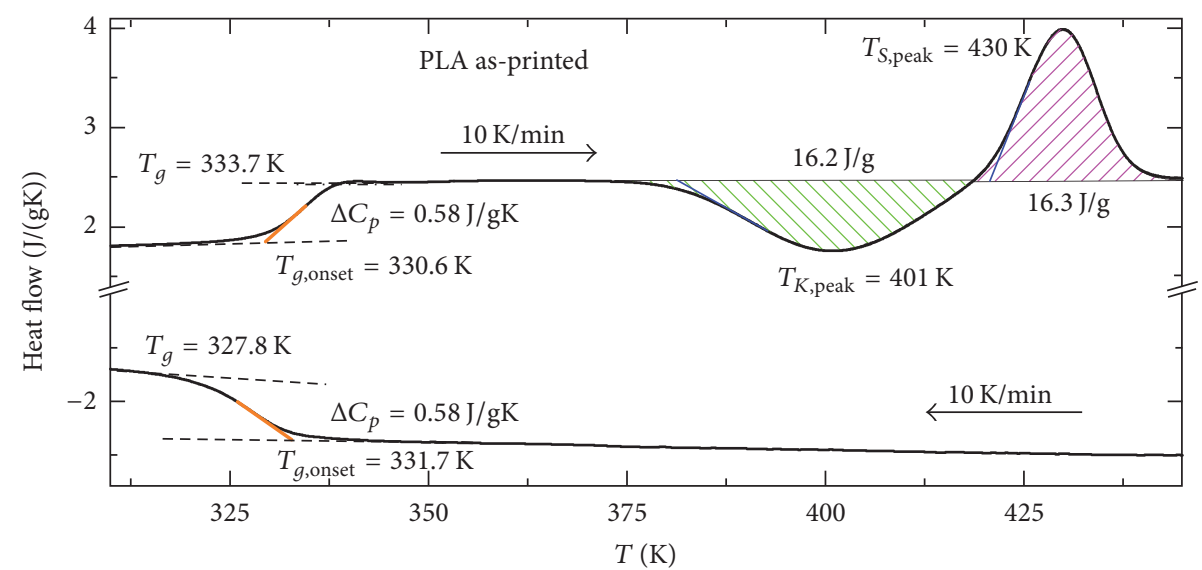

(a)

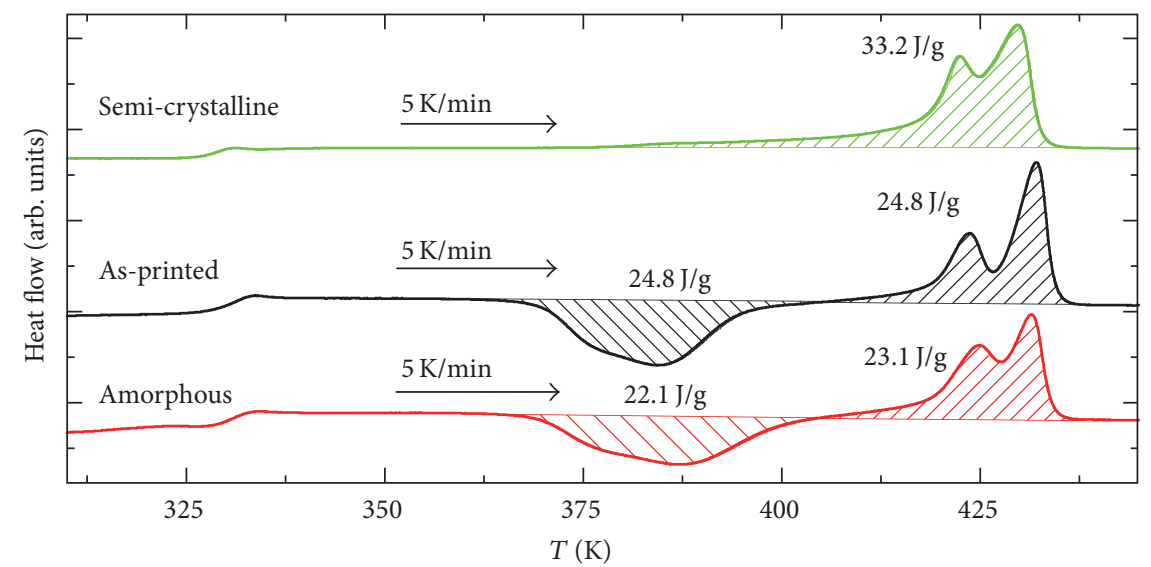

(b)

FIGURE 1: (a) Heat flow (endotherms up) of initial heating and subsequent cooling traces of PLA in the "as-printed" condition at a rate of $10 \mathrm{~K} / \mathrm{min}$. The glass transition temperature is determined using both the half step size $\left(T_{g}\right)$ and onset temperature ( $\left.T_{g, \text { Onset }}\right)$ methods. (b) DSC heating traces at a rate of $5 \mathrm{~K} / \mathrm{min}$, comparing PLA in the three conditions: semicrystalline (green), as-printed (black), and amorphous (red) conditions. The measurements are shifted vertically for clarity.

preparation. The stray-capacitance of this capacitor has been estimated based on its geometry, and this value " $\Delta C=$ $-1.1 \mathrm{pF}$ " is subtracted from all measurements, which have been conducted in this capacitor.

\section{Results and Discussion}

3.1. Differential Scanning Calorimetry of the Polymer PLA. Before discussing the electrical properties in detail, thorough analyses of the phase transitions are important. As described above, we used DSC to determine the glass transition temperatures $T_{g}$, the energy of the phase transitions, and the melting temperature. Furthermore, we exposed PLA to different cooling and heating rates. This allowed processing PLA in different modifications ranging from semicrystalline to purely amorphous state.

Figure 1(a) shows the specific heat flow versus temperature curve while initially heating and subsequently cooling PLA in the "as-printed" state. The measurement starts at ambient temperature using a heating rate of $10 \mathrm{~K} / \mathrm{min}$. A (cold) crystallization is an exothermic process as the value of specific heat shows a dip-like feature. As melting is an endothermic process a peak feature develops. The melting/crystallization temperature can be determined in two different ways: firstly, the peak-temperature and, secondly, the onset temperature. The entropy of a transition is determined by dividing the integrated peak-area in the temperature dependent heat flow plot by the temperature rate. A glass transition causes a change in heat capacity leading to a steplike feature in the temperature dependent heat flow. The onset method and the half height of this step-like feature are both used frequently to determine the glass transition temperature $T_{g}$. In our case we focus on the onset temperatures but also providing $T_{g}$ determined by the half-height method in Figure 1(a).

During the heating sequence three features are observed: the first one is the glass transition as a step-like anomaly at $331 \mathrm{~K}$ accompanied by a change in $\Delta C_{p}=0.58 \mathrm{~J} / \mathrm{gK} . T_{g}$ is determined from the onset temperatures of the heating curve. It is followed by a cold crystallization indicated by a dip-like minimum at about $401 \mathrm{~K}$ and an onset temperature of $382 \mathrm{~K}$. Finally, a peak feature at about $430 \mathrm{~K}$ (onset temperature $421 \mathrm{~K}$ ) denotes the melting of the crystalline phase of PLA. Inkinen et al. [28] reported a melting temperature 
of about $480 \mathrm{~K}$ for laboratory grade PLA. The discrepancy to our measured value originates from the chain length and additives of industrially produced PLA. Both effects result in a lower melting temperature. This points also towards a high molar mass of the PLA filament $(>100.000 \mathrm{~g} / \mathrm{mol})$ [29]. As expected, for PLA in the purely glassy phase, which is discussed below, the entropies of the cold crystallization $(16.2 \mathrm{~J} / \mathrm{g})$ and the melting $(16.3 \mathrm{~J} / \mathrm{g})$ are identical within the experimental uncertainty. That means "as-printed" PLA is most likely a pure glass below $331 \mathrm{~K}$. The subsequent cooling from the meltdown to ambient temperatures with a cooling rate of $10 \mathrm{~K} / \mathrm{min}$ confirms the result of Cao et al. [21] that this rate is enough to prevent crystallization of PLA. The values for these phase transitions, especially temperatures, agree well with the results from literature $[21,27,29,30]$. However, the glass transition temperature in literature varies about $\pm 5 \mathrm{~K}$, which most likely originates from the different stereoisomeric composition of (d,l)-lactide in PLA.

Figure 1(b) shows DSC heating curves for "as-printed" (black line), semicrystalline (green line), and purely amorphous PLA (red line). All samples were heated at a rate of $5 \mathrm{~K} / \mathrm{min}$. This rate was chosen to investigate the impact of heating rate on the crystalline fraction in PLA. The glass transition temperature $\left(T_{g} \approx 331 \mathrm{~K}\right)$ is almost the same for these three samples. The upper curve, which denotes the semicrystalline sample, showed no cold crystallization process. A cold crystallization can only evolve if the possibility for a further crystallization is given. For the preparation of the present semicrystalline sample we used a cooling rate of approximately $0.2 \mathrm{~K} / \mathrm{min}$, starting at a temperature above the melting point, and a subsequent tempering process for $10 \mathrm{~min}$ at $383 \mathrm{~K}$. This route allows reaching the maximum degree of crystallization in PLA, which is in the range of approximately $40 \%$ [26]. Thus, the semicrystalline sample exhibited only the glass transition and a double peak feature $\left(T_{p 1} \approx 422 \mathrm{~K}\right.$ and $T_{p 2} \approx 430 \mathrm{~K}$ ) with an enthalpy of $33 \mathrm{~J} / \mathrm{g}$ close to the melting point. This melting enthalpy is about twice the value of that for "as-printed" PLA heating at a rate of $10 \mathrm{~K} / \mathrm{min}$, allowing the assumption that for the printed PLA during cold crystallization a degree of crystalline phase in the order of $20 \%$ can be achieved.

On the other hand, the purely amorphous and the "asprinted" samples show a different behaviour. For both conditions, exceeding $T_{g}$ a cold crystallization occurs at about $370 \mathrm{~K}$ for "as-printed" and $368 \mathrm{~K}$ for amorphous PLA. A double peak feature due to the melting of the polymer follows. The enthalpies of cold crystallization and melting (approx. $25 \mathrm{~J} / \mathrm{g}$ for "as-printed" and $23 \mathrm{~J} / \mathrm{g}$ for "amorphous" PLA) are nearly identical, allowing the conclusion that both samples are below $T_{g}$ in a purely amorphous state. Compared to the DSC result of Figure 1(a) the enthalpy of "as-printed" PLA is about 1.5 times higher, which originates from an increased degree of crystalline phase (about 30\%) during heating with a rate of $5 \mathrm{~K} / \mathrm{min}$. Also, the melting feature exhibits two distinct maxima, which arise from two melting processes. The reason for that can be the lower heating rate of $5 \mathrm{~K} / \mathrm{min}$, leading to a separation of two melting processes. These two processes are based on different crystalline structures, which arise again from the low heating or cooling rate through the (cold) crystallization state [31]. Interestingly, for those two samples the peak maxima ("as-printed" PLA: $T_{p 1} \approx 423 \mathrm{~K}$ and $T_{p 2} \approx$ $432 \mathrm{~K}$; amorphous PLA: $T_{p 1} \approx 425 \mathrm{~K}$ and $T_{p 2} \approx 431 \mathrm{~K}$ ) and even the shapes of the peaks vary slightly. These deviations of the enthalpies and peak shapes of amorphous and the "as-printed" PLA originate from their mesoscopic structure formed during cooling from the melt. In case of printed PLA through a nozzle we expect a strongly anisotropic structure. For the amorphous PLA a more homogenous cooling without external mechanical stress was used. To clarify the real mesoscopic structure, optical polarization microscopy should be performed as shown for PLA in [31]. Now we address the question of whether the dielectric properties of PLA change, especially in the case of "as-printed" and amorphous state.

\subsection{Dielectric Properties}

3.2.1. Temperature Dependent Dielectric Properties of "AsPrinted" PLA. Figure 2 shows the temperature dependent dielectric constant $\varepsilon^{\prime}$ (a), dielectric loss $\varepsilon^{\prime \prime}$ (b), and the conductivity $\sigma^{\prime}$ (c) in a frequency range from $1 \mathrm{~Hz}$ to $3 \mathrm{GHz}$ for the "as-printed" PLA. The dielectric spectra are recorded while heating the sample at a rate of $0.2 \mathrm{~K} / \mathrm{min}$, starting at $150 \mathrm{~K}$. In the temperature dependent dielectric spectra typical signatures of orientational relaxations show up, giving rise to a frequency-dependent step-like increase in dielectric constant accompanied by a peak in dielectric loss. Relaxations have distinct frequency dependencies. Against this, structural changes, like cold crystallization, often become evident as a frequency-independent distinct step in a narrow temperature range for all dielectric quantities. Based on the DSC results, we discuss the dielectric properties, firstly, for temperatures below $T_{g}$, thus mainly focusing on dielectric properties in the glassy state. Secondly, we address the significant impact of increased dipolar dynamics above $T_{g}$ and the influence of cold crystallization on the dielectric properties.

For $T<T_{g}(331 \mathrm{~K})$ the dielectric constant in Figure 2(a) exhibits a marginal relaxation, which shows up as a smeared out step at low temperatures. This also gives rise to a broadened peak in $\varepsilon^{\prime \prime}$ (e.g., $210 \mathrm{~K}$ for $16 \mathrm{~Hz}$ ) and a slight reduction of the conductivity, for example, for $16 \mathrm{~Hz}$ between $150 \mathrm{~K}$ and $200 \mathrm{~K}$, depicted in Figures 2(b) and 2(c), respectively. Often reorientation of side chains [32] is the reason of such relaxations. However, PLA is ascribed to be a so-called type "A polymer" [33] that normally has no polar side chains. Thus, we conclude a Johari-Goldstein process [34] as the origin of the small relaxation, which is of minor interest for the technical applications due to the low temperatures and the marginal relaxation strength. PLA is applied as material for structural components or for electrical insulating purposes at ambient temperatures; for the latter the conductivity is an essential quantity. PLA in the "as-printed" state exhibits a $\sigma_{\mathrm{dc}}$, which varies from insulating $\left[<10^{-13}(\Omega \mathrm{cm})^{-1}\right]$ for low frequencies to an enhanced conductivity $\left[>10^{-5}(\Omega \mathrm{cm})^{-1}\right]$ for frequencies in the gigahertz regime. This is a typical behaviour for materials with localised charge carriers, for example, ionic impurities, which can be often found in amorphous materials. The mobility of these charges is based 


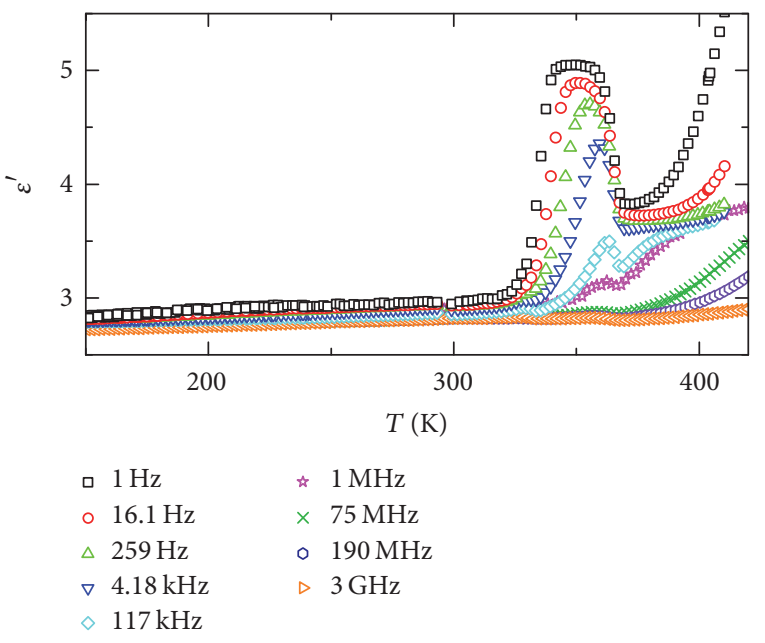

(a)

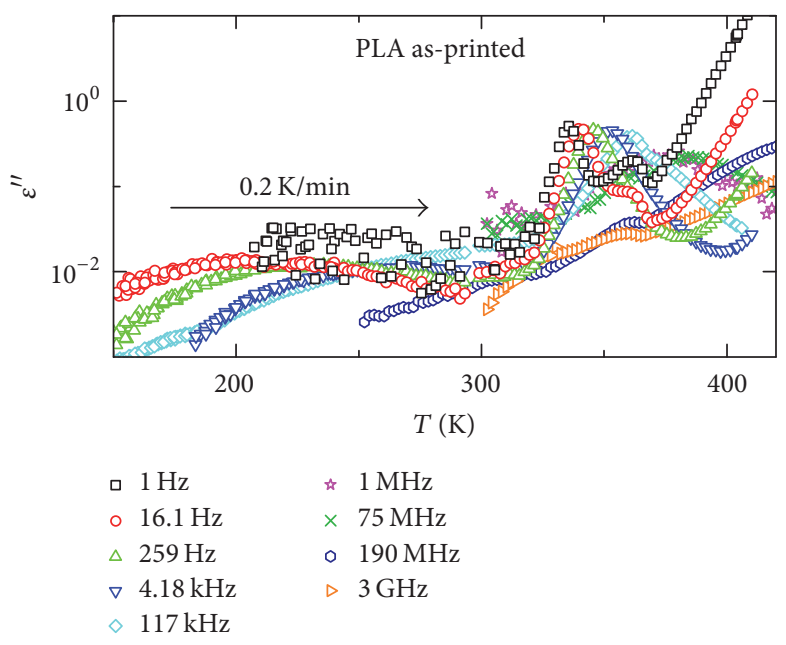

(b)

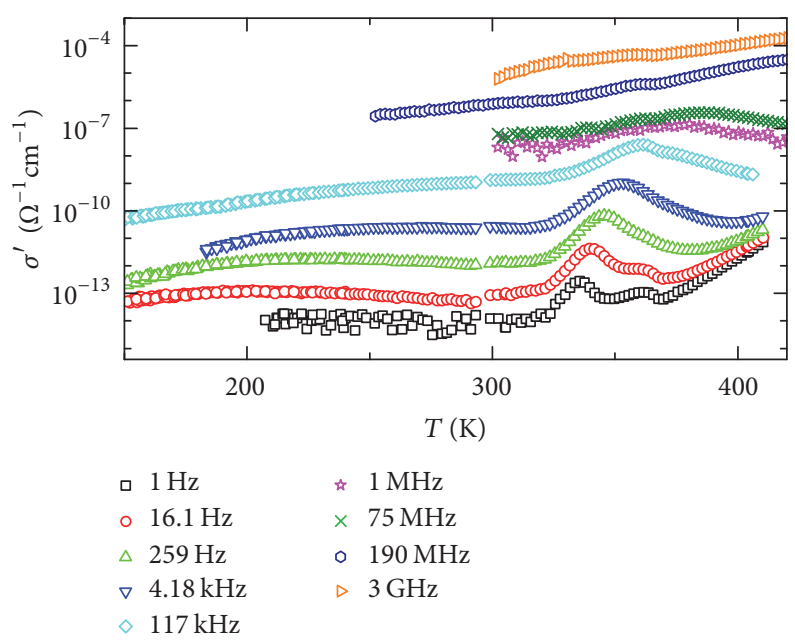

(c)

Figure 2: (a) Dielectric constant $\varepsilon^{\prime}$, (b) dielectric loss $\varepsilon^{\prime \prime}$, and (c) conductivity $\sigma^{\prime}$ measurements are displayed as functions of temperature for PLA in the as-printed condition. Various frequencies in a range of $1 \mathrm{~Hz}$ to $3 \mathrm{GHz}$ are shown during heating from $150 \mathrm{~K}$ to $420 \mathrm{~K}$ at a rate of $0.2 \mathrm{~K} / \mathrm{min}$. on hopping processes and increases especially when the temperature exceeds $T_{g}$.

For $T>T_{g}(331 \mathrm{~K})$ a significant frequency-dependent step-like behaviour is observed at about $336 \mathrm{~K}$ for $1 \mathrm{~Hz}$ increasing the dielectric constant from 2.9 to a plateau value at about 5 [cf. Figure 2(a)]. This feature shifts to higher temperatures with increasing frequencies. It is the main relaxation based on the reorientation of dipolar molecules, that is, chains of the polymer. Subsequently, at about $362 \mathrm{~K}$ the dielectric constant decreases nearly independently of frequency, for example, the $16 \mathrm{~Hz}$ curve down to a further almost static value of about 3.6. This decrease arises from the structural change of PLA during the cold crystallization. In particular, for frequencies above $117 \mathrm{kHz}$ the main relaxation process is shifted into the temperature regime of the cold crystallization, which shows up in Figure 2(a) as kink in $\varepsilon^{\prime}$ while the main relaxation develops. Ren et al. [33, 35] attribute the main relaxation to two processes: a sequential mode for $T<$ $350 \mathrm{~K}$ and a so-called "end-to-end" vector fluctuation for $350 \mathrm{~K}<T<390 \mathrm{~K}$. The latter describes the orientation of huge fragments of the polymer. However, it is also possible to explain the dielectric feature only using the so-called $\alpha$ relaxation, where the orientation of the molecules is partially hampered by the cold crystallization. This also results in the reduced plateau of the dielectric constant.

For higher temperatures $(T>380 \mathrm{~K})$ and low frequencies $(\nu<259 \mathrm{~Hz})$ an increase in $\varepsilon^{\prime}$ starts again pointing towards an interface polarization effect, which can be explained in terms of a Maxwell-Wagner (MW) relaxation process [36, 37]. All of the discussed features are accompanied by peaks or shoulders in the dielectric loss [cf. Figure 2(b)] as well as in the conductivity [cf. Figure 2(c)], for example, for the $1 \mathrm{~Hz}$ curve at $336 \mathrm{~K}$ and $362 \mathrm{~K}$. For higher temperatures, $T>400 \mathrm{~K}$, the lower frequencies $(\nu<4 \mathrm{kHz})$ exhibit the same temperature dependent behaviour (e.g., $1 \mathrm{~Hz}$ and $16.1 \mathrm{~Hz}$ ). This frequencyindependent increase in conductivity corresponds to the dcconductivity. As we use this conductivity to compare PLA with PLA mixed with IL, it should be noted that this dcconductivity is in the semicrystalline phase of PLA. Due to its low value, the trajectory of the dc-conductivity does not show up within the amorphous phase under the applied frequencies. The samples are only partly crystalline; thus we estimate the dc-conductivity of the amorphous phase in its proximity.

3.2.2. Frequency-Dependent Dielectric Properties of "AsPrinted" PLA. To investigate the main relaxation process in detail, Figure 3 depicts the frequency-dependent dielectric constant (a) and the dielectric loss (b) for temperatures above the glass transition ranging from $340 \mathrm{~K}$ to $410 \mathrm{~K}$. The lines are fits using only a Havriliak-Negami function [19] to describe the orientational relaxation process. This model originates from the Debye function describing the dielectric properties of a relaxation for noninteracting dipoles. However, dipole-dipole interactions and structural barriers necessitate a modification, which takes broadening and asymmetry of the Debye relaxation into account. The Havriliak-Negami (HN) function uses both parameters. For the polymer PLA normally a Cole-Davidson function should be sufficient to 


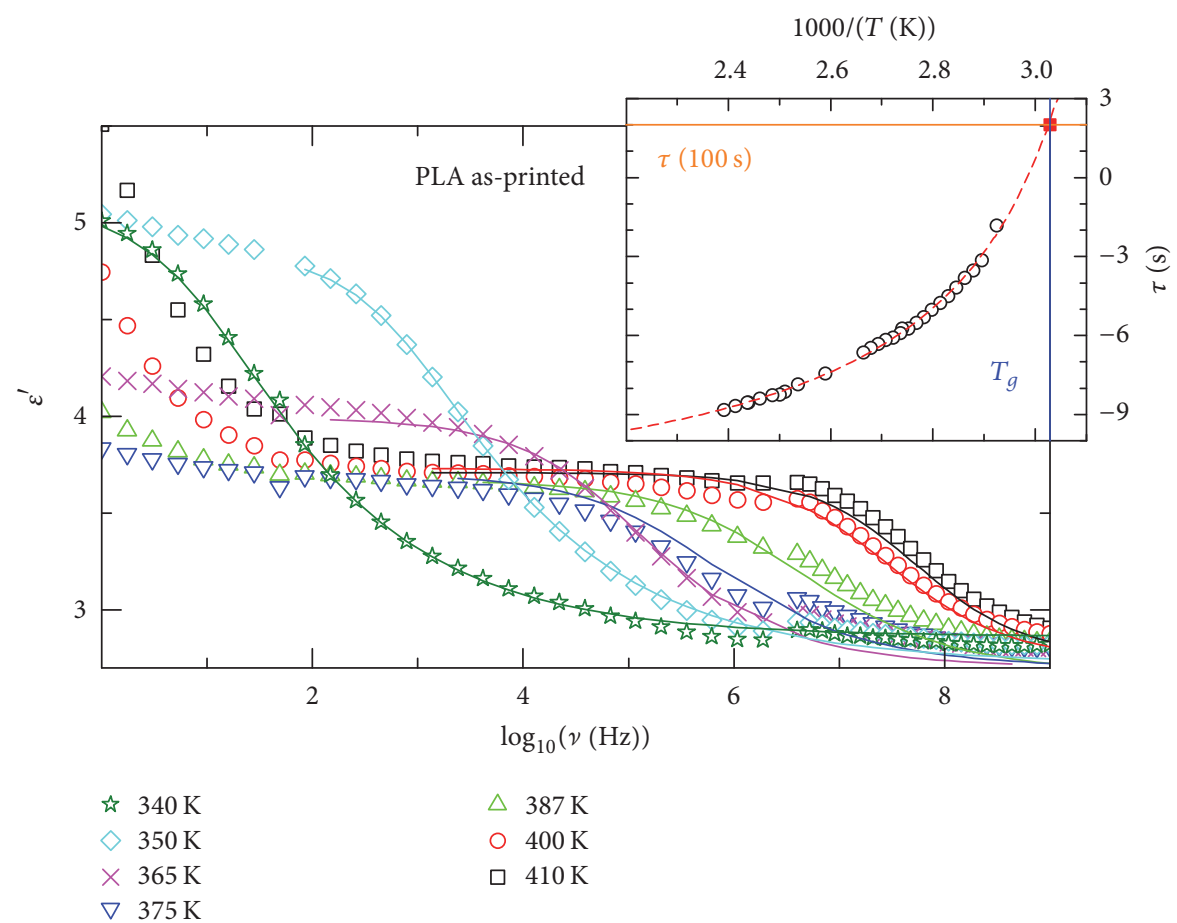

(a)

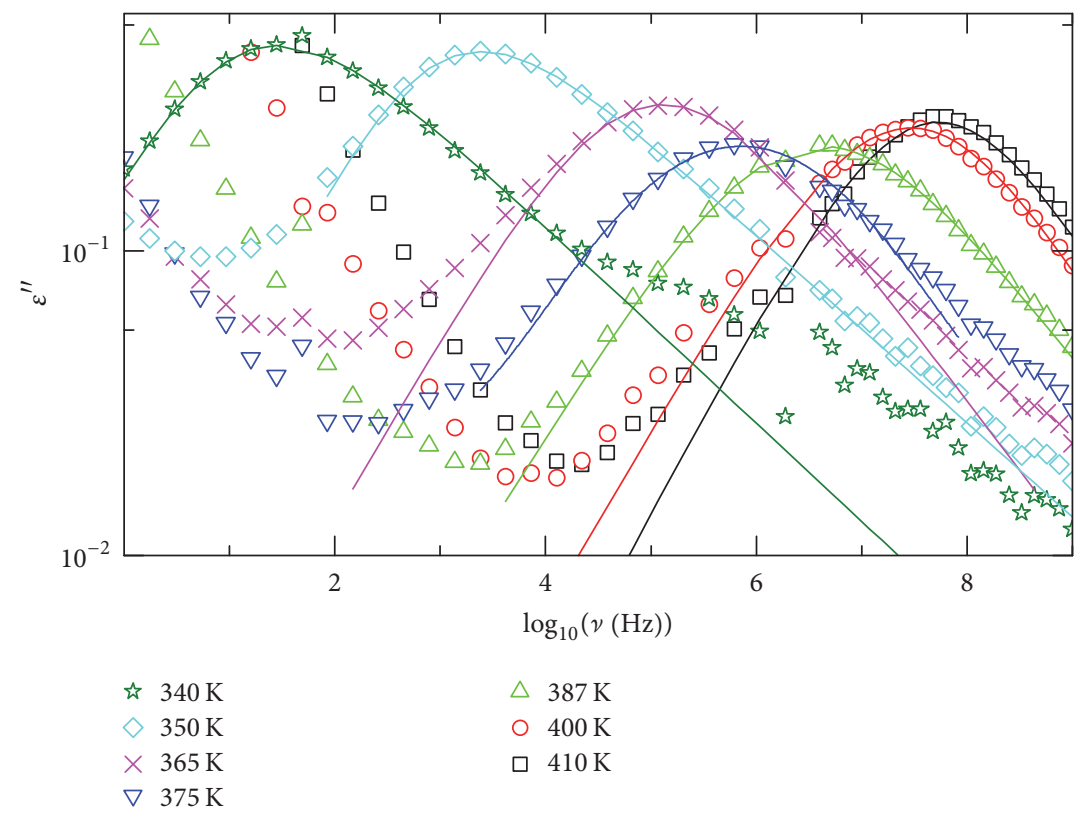

(b)

FIGURE 3: Dielectric constant $\varepsilon^{\prime}$ (a) and dielectric loss $\varepsilon^{\prime \prime}$ (b) of PLA in the as-printed condition; measurements are shown as functions of frequency for temperatures ranging from $340 \mathrm{~K}$ to $410 \mathrm{~K}$. Lines are fits according to Havriliak-Negami (HN) model. The inset shows the relaxations times according to the peak maxima of the dielectric loss in an Arrhenius representation fitted with a VFT function (dashed line). The glass transition temperature is indicated at $\tau(100 \mathrm{~s})$.

describe the main relaxation, taking only the asymmetry parameter into account. However, there is an additional need of the symmetric broadening parameter sufficiently fitting the spectra. The purification level of an industrially produced PLA is not exactly known, but it is reasonable to assume impurities, which are responsible for the distribution of relaxation times in the dielectric spectra.
As already discussed for Figure 2, the dielectric constant in Figure 3(a) shows a relaxation, which can be assigned to orientational polarization of PLA. The lines in Figure 2 are fits accounting only for the contribution of this relaxation. Again, for temperatures below the cold crystallization $(T<362 \mathrm{~K})$, the static dielectric constant, which is the low frequency plateau, reaches values of about 5 ; for $T>362 \mathrm{~K}$ the static 


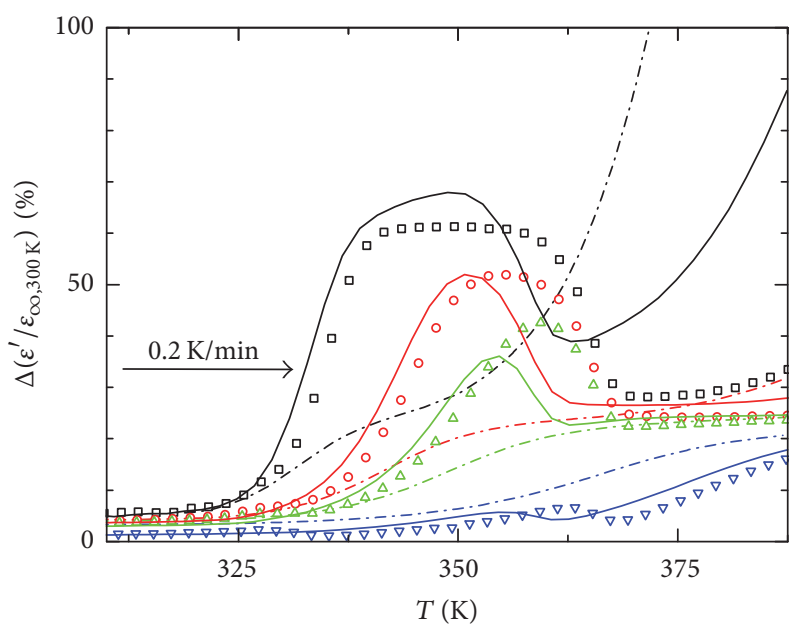

$\begin{array}{lll}\text { Semi-crystalline } & \text { As-printed } & \text { Amorphous } \\ ---1 \mathrm{~Hz} & \square 1 \mathrm{~Hz} & -1 \mathrm{~Hz} \\ ---259 \mathrm{~Hz} & \circ 259 \mathrm{~Hz} & -259 \mathrm{~Hz} \\ ---4.2 \mathrm{kHz} & \triangle 4.2 \mathrm{kHz} & -4.2 \mathrm{kHz} \\ ---1.1 \mathrm{MHz} & \nabla 1.1 \mathrm{MHz} & -1.1 \mathrm{MHz}\end{array}$

FIGURE 4: Comparison of the normalized (to $\varepsilon^{\prime}$ at $300 \mathrm{~K}$ ) dielectric constant $\varepsilon^{\prime}$ for PLA in the amorphous (solid lines), as-printed (closed symbols), and semicrystalline (dashes-dotted lines) state. The displayed result of the relative change in $\varepsilon^{\prime}$ is shown for the heating curve at a rate of $0.2 \mathrm{~K} / \mathrm{min}$ for various frequencies.

dielectric constant drops down to about 3.6. In particular, for $v<100 \mathrm{~Hz}$ and $T>400 \mathrm{~K}$ an onset of another dielectric relaxation develops, which is most likely a relaxation of Maxwell-Wagner type, originating from a so-called blocking electrode effect $[36,37]$. In addition, an excess intensity at the high frequency flank (cf. $340 \mathrm{~K}$ curve at about $10 \mathrm{MHz}$ ) denotes the already discussed Johari-Goldstein relaxation. The HN model describes the main relaxation, step in $\varepsilon^{\prime}$ (a) and peak in $\varepsilon^{\prime \prime}$ (b), quite well. The relaxation times $\tau(\mathrm{s})$, which are revealed by the temperature dependent peak position of $\varepsilon^{\prime \prime}$, are illustrated in an Arrhenius representation in the inset of Figure 3. The dashed line represents a Vogel-Fulcher-Tammann (VFT) function [38-41], which is extrapolated to $\tau(\mathrm{s})=100 \mathrm{~s}$. This point corresponds to the glass transition temperature $[40,42] T_{g}=330 \mathrm{~K}$ revealed from $\tau(\mathrm{s})$ and agrees well with $T_{g}$ measured from DSC. The Arrhenius representation also reveals that there is no significant change in temperature dependence of $\tau$ around the cold crystallization (cf. inset of Figure 3 around $2.8[1000 / \mathrm{K}]$ ). The VFT-formula smoothly fits both states. This implies only a single dipolar mechanism, which is contradictory to the two processes model proposed by Ren et al. $[33,35]$.

\subsubsection{Comparison of Dielectric Properties of PLA Modifica-} tions. For comparison of the different modifications of PLA, Figure 4 shows the temperature dependent relative change in dielectric constant (normalized to $\varepsilon^{\prime}$ at $300 \mathrm{~K}$ ) for selected frequencies, focusing on the dielectric properties around $T_{g}$. The "as-printed" and the amorphous sample exhibit an increase in $\Delta \varepsilon^{\prime}$ of about $65 \%$ for $T>T_{g}(331 \mathrm{~K})$. Subsequently, at the cold crystallization $\Delta \varepsilon^{\prime}$ drops down to about $25 \%$. For amorphous PLA this drop-down feature is shifted to a lower temperature (about $5 \mathrm{~K}$ ). Likewise, for DSC measurements a lower onset temperature for cold crystallization has been detected. In contrast, the dielectric properties of the semicrystalline sample only show an increase in $\varepsilon^{\prime}$, which arises from the enhanced dipolar dynamics of the glassy volume fraction above $T_{g}$. In addition, a MW feature shows up in the $1 \mathrm{~Hz}$ curves for the semicrystalline sample at $T>$ $345 \mathrm{~K}$ and for the amorphous sample at $T>360 \mathrm{~K}$. For the "as-printed" sample the MW feature is rather marginal, as the onset is hardly visible and can be assumed to be at about $380 \mathrm{~K}$ for the $1 \mathrm{~Hz}$ curve. Due to the fact that only the onset of the MW-relaxation is within the measured temperature regime, its origin is under debate. Most likely, the MW feature arises from an insulating surface layer at the electrode interfaces, based on the accumulation of ions causing a blocking layer effect. These thin insulating layers give rise to a high capacitance leading to a step in $\varepsilon^{\prime}$. The mobility of charge carriers often influences the temperature dependent onset of a MW feature, resulting in lower onset temperatures in the case of a higher mobility. It is still unclear why the mobility of charge carries in the "as-printed" is lower than in the amorphous sample, although both are quite similar from their overall dielectric behaviour. One could speculate that a different mesoscopic structure is formed during the printing process, causing an anisotropic conductivity, which is decreased perpendicular to the printing layers. Another explanation for the MW feature is an internal barrier layer. At the interface of crystalline and glassy fraction thin insulating layers can be formed. A detailed study of precisely prepared samples analysed with microscopic measurements and dielectric spectroscopy must be conducted to address the origin of this dielectric relaxation process and the shift in temperature. Altogether, the comparison of dielectric properties shows that the "as-printed" sample is quite similar to the amorphous rather than semicrystalline sample.

\subsection{Enhanced Conductivity of PLA Mixed with Ionic Liquid.} Finally, we demonstrate a route to prepare a more conductive PLA, which may be useful for $3 \mathrm{D}$ printing processes. Figure 5 shows that adding an ionic liquid to PLA increases the conductivity of the filament. In Figure 5(a) we compare the previously discussed conductivity spectra of "as-printed" PLA (open orange symbols) from industrial fabrication to samples of PLA in laboratory grade, which were mixed with the ionic liquid THTPT DE. However, as small amounts of additives can have a significant impact on structural, chemical, and physical properties of PLA, we analysed in addition a PLA sample, which was prepared in analogy to the mixed samples. The conductivity is in the same order of magnitude as the "as-printed" PLA (not shown). Thus, for the following the "as-printed" PLA is used as benchmark system. The PLA/IL samples were prepared, as described in Section 2, with 5 (blue symbols) and $10 \mathrm{wt} \%$ (open green symbols) of that IL. The dielectric spectra were recorded while heating the samples. At the lowest temperatures a marginal dielectric feature appears for both samples, for example, for $67 \mathrm{kHz}$ at $150 \mathrm{~K}$ (sample with $10 \mathrm{wt} \%$ ). The feature can be assigned to 


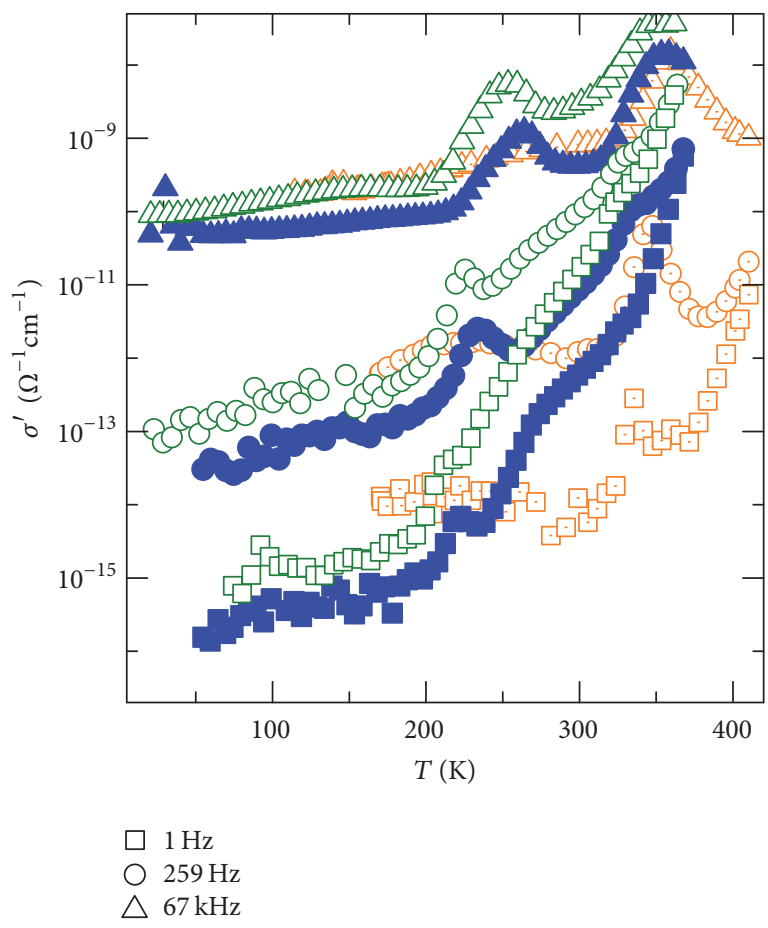

(a)

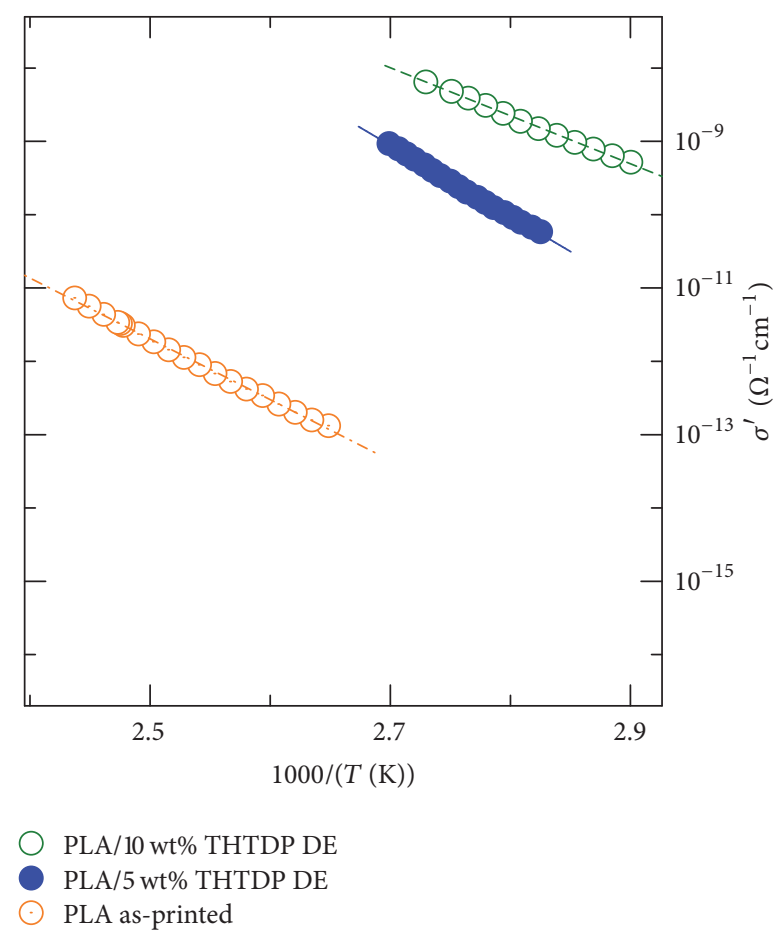

(b)

Figure 5: (a) Temperature dependent conductivity for selected frequencies of 5 and $10 \mathrm{wt} \%$ IL added PLA and "as-printed" PLA. (b) Arrhenius representation of evaluated dc-conductivities. Lines denote a thermally activated behaviour following an Arrhenius law.

a Johari-Goldstein relaxation. Interestingly, the temperature of that feature is strongly decreased compared to the "asprinted" sample, for which it occurs at $250 \mathrm{~K}$. This decrease implies an increase in the speed of the relaxation pointing towards an enhanced mobility of the molecules. At higher temperatures, around $220 \mathrm{~K}$, another feature emerges as a peak in the spectra. This relaxation is absent in the "asprinted" sample and thus based on dipoles introduced by the ionic liquid. Finally, at temperatures around $350 \mathrm{~K}$ for $67 \mathrm{kHz}$ a small shoulder resembles the main relaxation of the PLA. Interestingly, at a frequency of $67 \mathrm{kHz}$ the relaxations are almost at the same temperature, whereas for lower temperatures the spectra clearly diverge for the different samples. For $T>350 \mathrm{~K}$ the lower frequencies join the same temperature dependence for each sample, for example, $1 \mathrm{~Hz}$ and $259 \mathrm{~Hz}$ around $350 \mathrm{~K}$ for the sample with $10 \mathrm{wt} \%$. This superposition implies frequency-independence that represents the dc-conductivity of those samples, which is a key quantity for applications.

In Figure 5(b) we compare the evaluated dc-conductivities of the 5 and $10 \mathrm{wt} \%$ IL mixed PLA with the "as-printed" PLA (after cold crystallization) in an Arrhenius representation. Based on the strongly enhanced conductivities of PLA mixed with the IL, $\sigma_{\mathrm{dc}}$ was measured in a different temperature range. The slopes of the linear fits [lines in Figure 5(b)] are representing the activation energy in an Arrhenius law. For PLA "as-printed" and PLA/IL 10 wt\% we assume approximately the same value, but for PLA/IL $5 \mathrm{wt} \%$ it is increased. An extrapolation of the linear behaviour to $370 \mathrm{~K}$ (Figure 5(b) at $2.7[1000 / \mathrm{K}]$ ) for all samples reveals an increase in $\sigma_{\mathrm{dc}}$ in the order of 4 decades for PLA/IL $5 \mathrm{wt} \%$ and 5 decades for PLA/IL $10 \mathrm{wt} \%$, respectively. Thus, adding ionic liquids seems to be an appropriate way to modify the conductivity of PLA. To implement these substances as filament or droplet-inkjet for additive manufacturing a thorough rheology study has to be performed, which is not in the scope of the present manuscript. Furthermore, the mechanical properties can be modified in such a way that a filament fabrication is impossible. The observed minor change in structural $T_{g}$ points towards only a moderate variation in viscosity. Again mechanical studies should be conducted to clarify the structure-properties relationship.

\section{Conclusion}

In this manuscript, we analysed in detail the transition temperatures and the dielectric properties of polylactic acid, which was printed via fused filament fabrication in a lowbudget $3 \mathrm{D}$ printer. These properties are compared to precisely prepared samples of semicrystalline and amorphous PLA. The dielectric properties of "as-printed" PLA are similar to the amorphous ones rather than the semicrystalline allowing good insulating properties below the glass transition temperature. The dielectric analyses reveal dipolar relaxation processes and the significant change in dielectric properties during cold crystallization. It seems that the plateau of the main relaxation is proportional to the degree of crystalline phase. Interestingly, DSC and dielectric measurements reveal that the mesoscopic structure of "as-printed" PLA, which is most likely influenced by the feeding mechanism of the $3 \mathrm{D}$ 
printer, also influences cold crystallization and maybe even the mobility or number of charge carriers. The latter shows up as a change in the onset temperature of a Maxwell-Wagner type relaxation process. We already used these materials for lab purposes, for example, as spacer disc for dielectric analysis of liquid samples. In addition, we show as an example for possible future functionalization of PLA that adding a low amount of the ionic liquid THTPT DE strongly increases the conductivity of PLA. However, a focused rheology study has to be performed and further PLA compositions should be analysed. The present "case study" shows a possible route to make PLA also suitable for rapid fabrication of electronic components.

\section{Conflicts of Interest}

The authors declare that there are no conflicts of interest regarding the publication of this paper.

\section{Acknowledgments}

The authors thank Robert Horny from the University of Augsburg for partially performing the DSC measurements and Stefanie Jung for assisting in preparing and measuring the IL/PLA mixtures. This research was supported by the Federal Ministry of Education and Research via Project 03EK3015 and by the Bavarian State Ministry of Education, Science and the Arts in form of the Graduate Program "Resource Strategy Concepts for Sustainable Energy Systems" at the Institute of Materials Resource Management (MRM) of the University of Augsburg, Germany.

\section{References}

[1] G. E. Ryan, A. S. Pandit, and D. P. Apatsidis, "Porous titanium scaffolds fabricated using a rapid prototyping and powder metallurgy technique," Biomaterials, vol. 29, no. 27, pp. 36253635, 2008.

[2] H. N. Chia and B. M. Wu, "Recent advances in 3D printing of biomaterials," Journal of Biological Engineering, vol. 9, 4 pages, 2015.

[3] C. Zhang, N. C. Anzalone, R. P. Faria, and J. M. Pearce, "Opensource 3D-printable optics equipment," PLoS ONE, vol. 8, no. 3, Article ID e59840, 2013.

[4] G. Comina, A. Suska, and D. Filippini, "Low cost lab-on-a-chip prototyping with a consumer grade 3D printer," Lab on a Chip - Miniaturisation for Chemistry and Biology, vol. 14, no. 16, pp. 2978-2982, 2014.

[5] S. K. Moon, Y. E. Tan, J. Hwang, and Y.-J. Yoon, "Application of 3D printing technology for designing light-weight unmanned aerial vehicle wing structures," International Journal of Precision Engineering and Manufacturing - Green Technology, vol. 1, no. 3, pp. 223-228, 2014.

[6] S. Pandey, B. Gupta, and A. Nahata, "Terahertz plasmonic waveguides created via 3D printing," Optics Express, vol. 21, no. 21, pp. 24422-24430, 2013.

[7] M. D. Symes, P. J. Kitson, J. Yan et al., "Integrated 3D-printed reactionware for chemical synthesis and analysis," Nature Chemistry, vol. 4, no. 5, pp. 349-354, 2012.
[8] C. Zhang, B. Wijnen, and J. M. Pearce, "Open-Source 3-D Platform for Low-Cost Scientific Instrument Ecosystem," Journal of Laboratory Automation, vol. 21, no. 4, pp. 517-525, 2016.

[9] J. M. Pearce, "Return on investment for open source scientific hardware development," Science and Public Policy, vol. 43, no. 2, pp. 192-195, 2016.

[10] D. T. Pham and R. S. Gault, "A comparison of rapid prototyping technologies," International Journal of Machine Tools and Manufacture, vol. 38, no. 10-11, pp. 1257-1287, 1998.

[11] P. S. P. Poh, M. P. Chhaya, F. M. Wunner et al., "Polylactides in additive biomanufacturing," Advanced Drug Delivery Reviews, vol. 107, pp. 228-246, 2016.

[12] R. Auras, B. Harte, and S. Selke, "An overview of polylactides as packaging materials," Macromolecular Bioscience, vol. 4, no. 9, pp. 835-864, 2004.

[13] L.-T. Lim, R. Auras, and M. Rubino, "Processing technologies for poly(lactic acid)," Progress in Polymer Science (Oxford), vol. 33 , no. 8, pp. 820-852, 2008.

[14] A. Södergård and M. Stolt, "Properties of lactic acid based polymers and their correlation with composition," Progress in Polymer Science (Oxford), vol. 27, no. 6, pp. 1123-1163, 2002.

[15] S. J. Leigh, R. J. Bradley, C. P. Purssell, D. R. Billson, and D. A. Hutchins, "A Simple, Low-Cost Conductive Composite Material for 3D Printing of Electronic Sensors," PLoS ONE, vol. 7, no. 11, Article ID e49365, pp. 1-6, 2012.

[16] E. Zuza, J. M. Ugartemendia, A. Lopez, E. Meaurio, A. Lejardi, and J.-R. Sarasua, "Glass transition behavior and dynamic fragility in polylactides containing mobile and rigid amorphous fractions," Polymer, vol. 49, no. 20, pp. 4427-4432, 2008.

[17] M. Kanchanasopa and J. Runt, "Broadband dielectric investigation of amorphous and semicrystalline L-lactide/meso-lactide copolymers," Macromolecules, vol. 37, no. 3, pp. 863-871, 2004.

[18] J. Ren and K. Adachi, "Dielectric relaxation in blends of amorphous poly(DL-lactic acid) and semicrystalline poly(L-lactic acid)," Macromolecules, vol. 36, no. 14, pp. 5180-5186, 2003.

[19] J. D. Badia, L. Monreal, V. Sáenz De Juano-Arbona, and A. Ribes-Greus, "Dielectric spectroscopy of recycled polylactide," Polymer Degradation and Stability, vol. 107, pp. 21-27, 2014.

[20] S. Hikosaka, H. Ishikawa, and Y. Ohki, "Effects of crystallinity on dielectric properties of poly(L-lactide)," Electronics and Communications in Japan, vol. 94, no. 7, pp. 1-8, 2011.

[21] X. Cao, A. Mohamed, S. H. Gordon, J. L. Willett, and D. J. Sessa, "DSC study of biodegradable poly(lactic acid) and poly(hydroxy ester ether) blends," Thermochimica Acta, vol. 406, no. 1-2, pp. 115-127, 2003.

[22] G. W. Ehrenstein, Polymer Werkstoffe, Carl Hanser Verlag GmbH \& Co. KG, München, Germany, 2011.

[23] K. I. Park and M. Xanthos, "A study on the degradation of polylactic acid in the presence of phosphonium ionic liquids," Polymer Degradation and Stability, vol. 94, no. 5, pp. 834-844, 2009.

[24] K. Park, J. U. Ha, and M. Xanthos, "Ionic liquids as plasticizers/lubricants for polylactic acid," Polymer Engineering and Science, vol. 50, no. 6, pp. 1105-1110, 2010.

[25] P. Wasserscheid and W. Keim, "Ionic Liquids-New 'Solutions' for Transition Metal Catalysis," Angewandte Chemie, vol. 39, no. 21, pp. 3772-3789, 2000.

[26] H. Cai, V. Dave, R. A. Gross, and S. P. McCarthy, "Effects of physical aging, crystallinity, and orientation on the enzymatic degradation of poly(lactic acid)," Journal of Polymer Science Part B: Polymer Physics, vol. 34, no. 16, pp. 2701-2708, 1996. 
[27] P. Sippel, V. Dietrich, D. Reuter et al., "Impact of water on the charge transport of a glass-forming ionic liquid," Journal of Molecular Liquids, vol. 223, pp. 635-642, 2016.

[28] S. Inkinen, M. Hakkarainen, A.-C. Albertsson, and A. Södergård, "From lactic acid to poly(lactic acid) (PLA): characterization and analysis of PLA and its precursors," Biomacromolecules, vol. 12, no. 3, pp. 523-532, 2011.

[29] P. Sanglard, V. Adamo, J.-P. Bourgeois, T. Chappuis, and E. Vanoli, "Poly(lactic acid) synthesis and characterization," Chimia International Journal for Chemistry, vol. 66, no. 12, pp. 951954, 2012.

[30] J. K. Jeszka, L. Pietrzak, M. Pluta, and G. Boiteux, "Dielectric properties of polylactides and their nanocomposites with montmorillonite," Journal of Non-Crystalline Solids, vol. 356, no. 11-17, pp. 818-821, 2010.

[31] J. Mijović and J.-W. Sy, "Molecular dynamics during crystallization of poly(L-lactic acid) as studied by broad-band dielectric relaxation spectroscopy," Macromolecules, vol. 35, no. 16, pp. 6370-6376, 2002.

[32] A. R. Brás, P. Malik, M. Dionísio, and J. F. Mano, "Influence of crystallinity in molecular motions of poly(L-lactic acid) investigated by dielectric relaxation spectroscopy," Macromolecules, vol. 41, no. 17, pp. 6419-6430, 2008.

[33] J. Ren, O. Urakawa, and K. Adachi, "Dielectric study on dynamics and conformation of poly(D,L-lactic acid) in dilute and semi-dilute solutions," Polymer, vol. 44, no. 3, pp. 847-855, 2003.

[34] C. P. Johari and M. Goidstein, "Viscous liquids and the glass transition. II. Secondary relaxations in glasses of rigid molecules," The Journal of Chemical Physics, vol. 53, no. 6, pp. 23722388, 1970.

[35] J. Ren, O. Urakawa, and K. Adachi, "Dielectric and viscoelastic studies of segmental and normal mode relaxations in undiluted poly(d,l-lactic acid)," Macromolecules, vol.36, no. 1, pp. 210-219, 2003.

[36] P. Lunkenheimer, S. Krohns, S. Riegg, S. G. Ebbinghaus, A. Reller, and A. Loidl, "Colossal dielectric constants in transitionmetal oxides," European Physical Journal: Special Topics, vol. 180, no. 1, pp. 61-89, 2009.

[37] S. Emmert, M. Wolf, R. Gulich et al., "Electrode polarization effects in broadband dielectric spectroscopy," European Physical Journal B, vol. 83, no. 2, pp. 157-165, 2011.

[38] H. Vogel, "The temperature dependence law of the viscosity of fluids," Physikalische Zeitschrift, vol. 22, pp. 645-646, 1921.

[39] G. S. Fulcher, "Analysis of Recent Measurements of the Viscosity of Glasses," Journal of the American Ceramic Society, vol. 75, no. 5, pp. 1043-1055, 1992.

[40] C. A. Angell, "Strong and fragile liquids," in Relaxations in Complex Systems, K. L. Ngai and G. B. Wright, Eds., pp. 3-11, NRL, Washington, DC, USA, 1985.

[41] G. Tammann and W. Hesse, "Die abhängigkeit der viskosität von der temperatur bei unterkühlten flüssigkeiten," Zeitschrift für Anorganische und Allgemeine Chemie, 1927.

[42] P. Sippel, P. Lunkenheimer, S. Krohns, E. Thoms, and A. Loidl, "Importance of liquid fragility for energy applications of ionic liquids," Scientific Reports, vol. 5, Article ID 13922, p. 13922, 2015. 

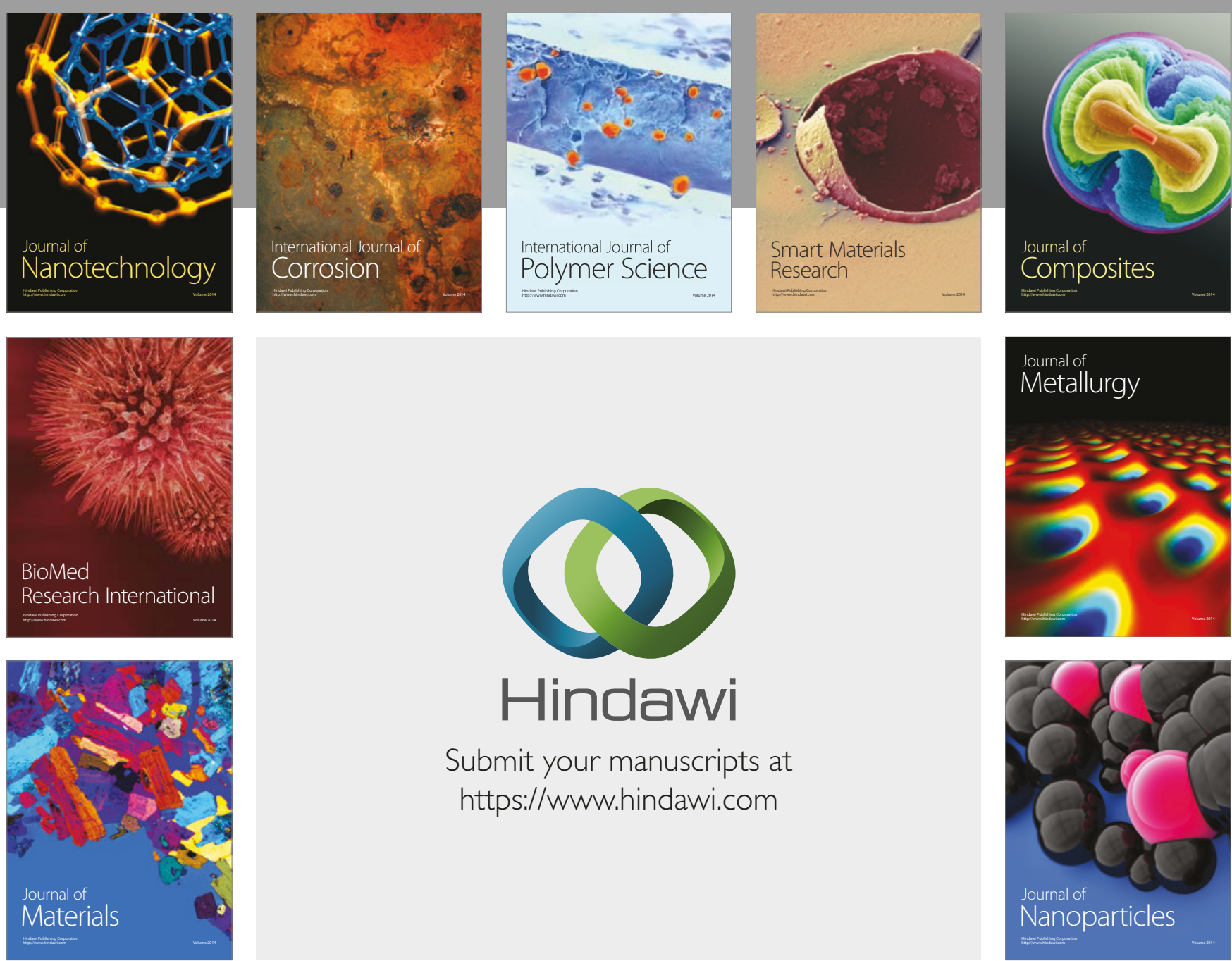

\section{Hindawi}

Submit your manuscripts at

https://www.hindawi.com
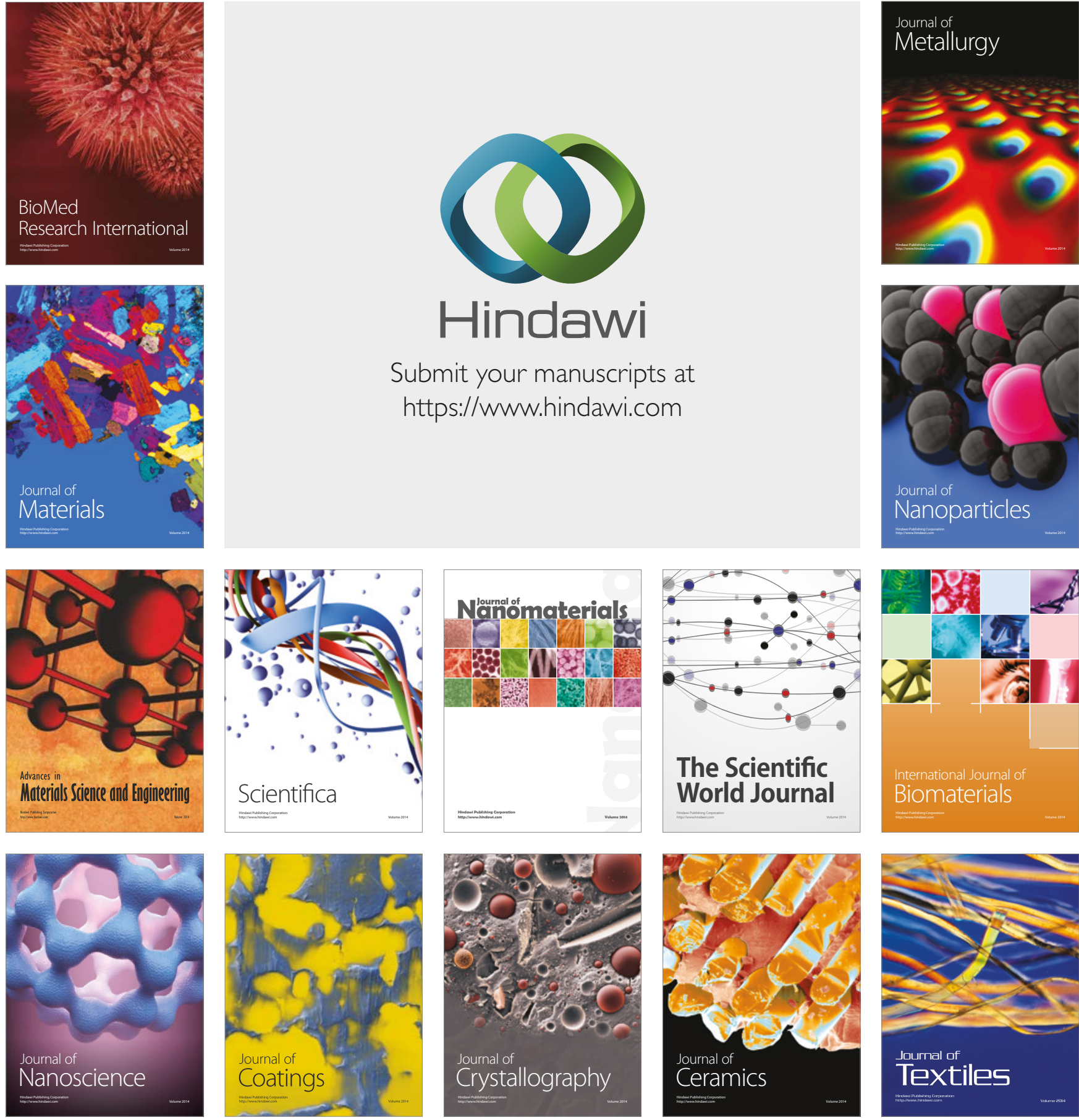

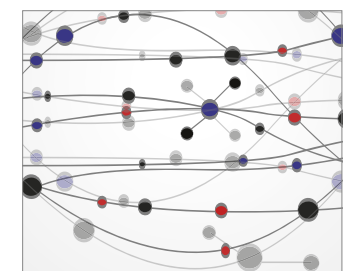

The Scientific World Journal
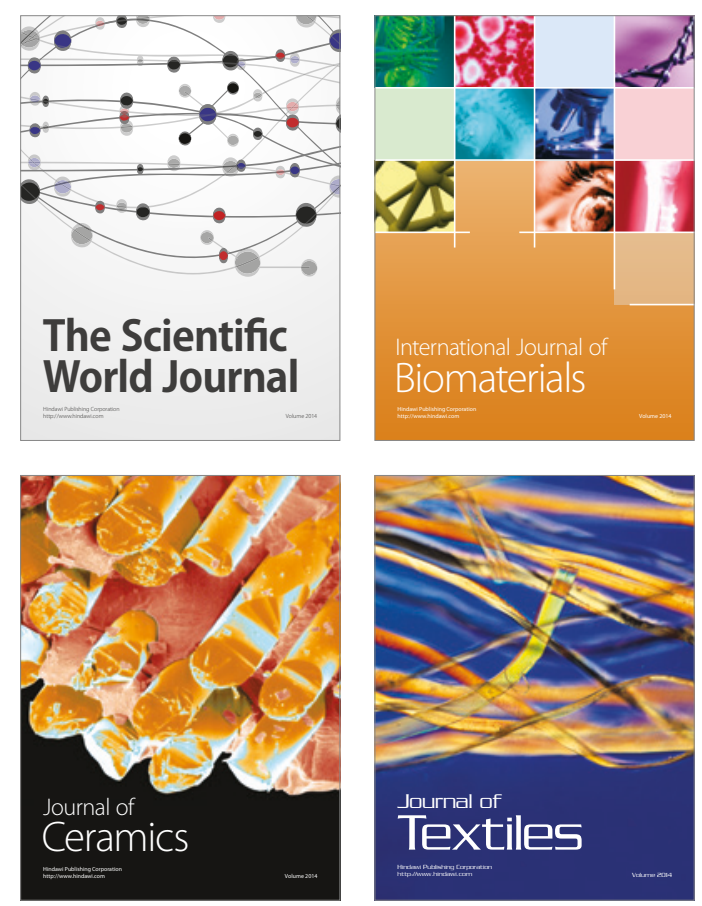